\title{
ERROR ANALYSIS OF STAGGERED FINITE DIFFERENCE FINITE VOLUME SCHEMES ON UNSTRUCTURED MESHES
}

\author{
QINGSHAN CHEN
}

\begin{abstract}
This work combines the consistency in lower-order differential operators with external approximations of functional spaces to obtain error estimates for finite difference finite volume schemes on unstructured non-uniform meshes. This combined approach is first applied to the one-dimensional elliptic boundary value problem on non-uniform meshes, and a first-order convergence rate is obtained, which agrees with the results previously reported. The approach is also applied to the staggered MAC scheme for the two-dimensional incompressible Stokes problem on unstructured meshes. A first-order convergence rate is obtained, which improves over a previously reported result in that it also holds on unstructured meshes. For both problems considered in this work, the convergence rate is one order higher on meshes satisfying special requirements.
\end{abstract}

\section{INTRODUCTION}

Staggered finite difference finite volume schemes (FDFV) place scalar variables and vectorial variables at different grid points in a staggered pattern. They inherit all the desirable conservative properties from the finite volume method. The staggered placement of the scalar and vectorial variables also render the schemes particularly suitable for transport phenomena. A classical staggered scheme is the famous Marker-and-Cell (MAC) scheme proposed by Harlow and Welch $([20])$. The MAC scheme is considered the method of choice for incompressible flows (see a comprehensive treatment of this topic by Wesseling ([27]). Since its introduction, it has also been argued that the MAC scheme is suitable for flows of all speeds $([18,19])$. A variant of the MAC scheme, the so-called C-grid scheme, has enjoyed wide popularity in the ocean and atmosphere modeling community for its superior performance in resolving the inertial-gravity waves $([2,[26,23])$. See [6, 4] for additional variants of the C-grid scheme.

Date: December 2, 2016.

2010 Mathematics Subject Classification. 65N06, 65N08, 65N12, 65N22, 76D07.

Key words and phrases. staggered-grid, MAC, incompressible Stokes, finite difference, finite volume, unstructured meshes, a priori error estimate.

This work is in part supported by a grant from the Simons Foundation (\#319070 to Qingshan Chen). 
Compared to the finite element method, the analysis of the finite volume method is less abundant, likely due to the lack of a natural variational framework, as finite volume schemes are typically not written in the variational form. However, due to the popularity of the finite volume methods in engineering and geophysical applications, theoretical analysis of these methods to determine their stability, convergence and accuracy is much needed. Through a mapping from the trial function space to the test function space, Li et al ([21]) cast non-staggered generalized finite difference schemes into a Galerkin variational framework, where the error analysis can be performed in the same way as for the finite element methods ([7]). Temam and coauthors employ functional analytical tools to study the convergence of nonstaggered finite volume schemes on structured and quasi-structured meshes ([14, 1, 15, 16]), by constructing external approximations of function spaces ([3]) and transforming the schemes into the variational forms. Chen ([5]) extends this approach to staggered finite difference finite volume schemes on unstructured meshes. Relying heavily on functional analytical tools, but without explicit approximations of functional spaces, Eymard et al ([13]) study the convergence and accuracy for non-staggered finite volume methods on structured and unstructured meshes. Droniou et al ([8]) establishes the convergence of a class of gradient schemes written in the discrete variational formulations.

The current work is concerned with the error analysis of staggered finite volume schemes on unstructured meshes. As highlighted by Eymard et al ([13]), a major hurdle to deriving error estimates for finite volume scheme is the fact that finite volume schemes are often not consistent with the differential equations they are approximating. The authors show that, actually, consistency in approximations of the fluxes is enough for error estimates in many cases. The authors apply this idea to derive error estimates for non-staggered finite volume schemes for linear elliptic and parabolic problems. For scalar linear hyperbolic problems, the finite volume schemes are consistent with the continuous equation, and their error estimates can be handled in the same way as the finite difference schemes. Error estimates for staggered finite volume schemes are scarce. Nicolaides ([22]) proves that the MAC scheme for the incompressible Stokes problem on a rectangular mesh is first-order accurate in both $L^{2}$ and $H^{1}$ norm.

The main thrust of this work is to show that, in the spirit of [13, consistency in lower-order differential operators, such as derivative or curl, is sufficient for error estimates in many cases. Here, we combine the consistency in lower-order operators with the external approximation framework to derive error estimates for staggered FDFV schemes. To illustrate how this combined approach works, we first apply it to a simple one-dimensional elliptic problem, and recover the results already presented in [13]. Then, in Section 3, we apply the combined approach to the MAC scheme for the Stokes problem on unstructured meshes. We show that the scheme is first-order accurate in both $L^{2}$ and $H^{1}$ norms, and in certain special cases, including 
the rectangular meshes, the scheme is second-order accurate under both $L^{2}$ and $H^{1}$ norms.

\section{The OnE-Dimensional ELLIPTIC PROBLEM}

We consider the one-dimensional elliptic problem

$$
\begin{aligned}
& -u_{x x}=f, \quad 0<x<1, \\
& u(0)=u(1)=0 .
\end{aligned}
$$

We define the function spaces

$$
\begin{aligned}
V & =H_{0}^{1}(0,1), \\
V^{\prime} & =H^{-1}(0,1) .
\end{aligned}
$$

For $u, v \in V$, we define the bilinear operator

$$
a(u, v)=\left(u_{x}, v_{x}\right) .
$$

Here, $(\cdot, \cdot)$ denotes the inner product of the $L^{2}(0,1)$ space. The weak formulation of the elliptic problem (2.1)-2.2 can now be stated:

For each $f \in V^{\prime}$, find $u \in V$ such that

$$
a(u, v)=\langle f, v\rangle, \quad \forall v \in V .
$$

If $u$ is a classical solution of the elliptic problem (2.1)-(2.2), then $u$ satisfies (2.3) as well. In this sense, (2.3) is a weak formulation of $2.1-(2.2)$. The existence and uniqueness of a solution to the weak problem is now part of the classical elliptic PDE theory, which is based on the fact that the bilinear operator $a(\cdot, \cdot)$ is coercive. The classical theory also provides that, if the right-hand side forcing $f$ is smooth, then the solution is also smooth.

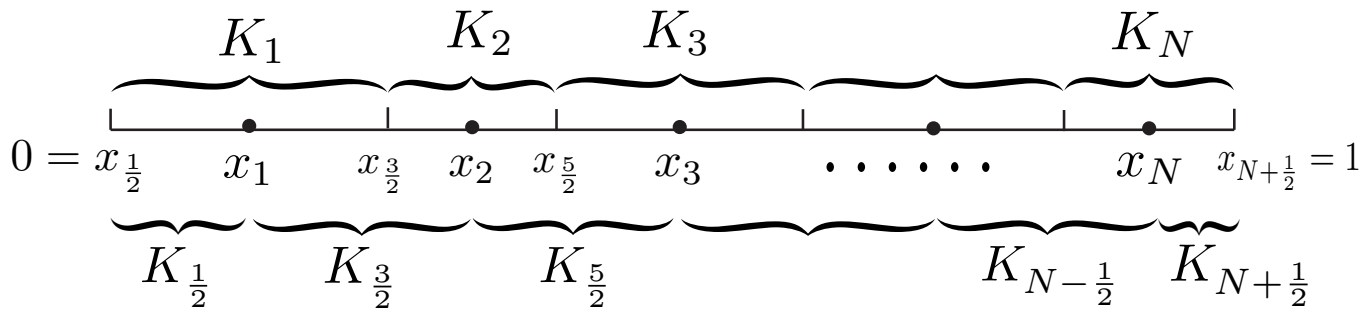

FiguRE 1. One-dimensional non-uniform mesh

\subsection{External approximation of $V$ and the finite volume scheme.} The interval $[0,1]$ is partitioned into $N$ subintervals $K_{i}, 1 \leq i \leq N$, which are centered at $x_{i}$, and have lengths of $h_{i}$ (Figure 1). The duals of $K_{i}$ are $K_{i+\frac{1}{2}}, 0 \leq i \leq N$, with the corresponding centers $x_{i+\frac{1}{2}}$, and lengths $h_{i+\frac{1}{2}}$. Here the term "center" is used in its liberal sense; $x_{i}$ (resp. $x_{i+\frac{1}{2}}$ ) is not 
necessarily the midpoint of $K_{i}$ (resp. $K_{i+\frac{1}{2}}$ ). The formal notations about the mesh are given below:

$$
\begin{aligned}
& x_{i} \in K_{i}=\left[x_{i-\frac{1}{2}}, x_{i+\frac{1}{2}}\right], \quad 1 \leq i \leq N \\
& x_{i+\frac{1}{2}} \in K_{i+\frac{1}{2}}=\left[x_{i}, x_{i+1}\right], \quad 1 \leq i \leq N-1 \\
& x_{\frac{1}{2}} \in K_{\frac{1}{2}}=\left[x_{\frac{1}{2}}, x_{1}\right], \quad x_{N+\frac{1}{2}} \in K_{N+\frac{1}{2}}=\left[x_{N}, x_{N+\frac{1}{2}}\right], \\
& h_{i}=\left|K_{i}\right|, \quad 1 \leq i \leq N, \\
& h_{i+\frac{1}{2}}=\left|K_{i+\frac{1}{2}}\right|, \quad 0 \leq i \leq N .
\end{aligned}
$$

We are interested in non-uniform meshes, and thus the mesh sizes $h_{i}$ and $h_{i+\frac{1}{2}}$ are non-constant. However, for regularity reasons, we assume that the ratio between the largest mesh size and the smallest mesh size is bounded. More precisely, we assume that, for each mesh, there exists $h>0$ such that

$$
m h<h_{i}, h_{i+\frac{1}{2}}<M h,
$$

where $m$ and $M$ are constants that are independent of the meshes. In what follows, each mesh is represented by a subscript ${ }_{h}$, and the total set of admissible meshes is designated as $\mathcal{H}$.

We let

$$
F=L^{2}(0,1) \times L^{2}(0,1) .
$$

We define an isomorphism $\Pi$ from $V$ into $F$ as

$$
\Pi u=\left(u, u_{x}\right) \in F, \quad \forall u \in V,
$$

where $u_{x}$ designates the first derivative of the function. For each $h \in \mathcal{H}$, the space $V$ is approximated by $V_{h}$ defined on the primary mesh and $\widetilde{V}_{h}$ defined on the dual mesh,

$$
\begin{aligned}
& V_{h}=\left\{u_{h}=\sum_{i=1}^{N} u_{i} \chi_{i}\right\}, \\
& \widetilde{V}_{h}=\left\{\widetilde{u}_{h}=\sum_{i=0}^{N} \widetilde{u}_{i+\frac{1}{2}} \chi_{i+\frac{1}{2}}\right\} .
\end{aligned}
$$

Here, $\chi_{i}$ and $\chi_{i+\frac{1}{2}}$ are the characteristic functions defined on the mesh cells $K_{i}$ and $K_{i+\frac{1}{2}}$, respectively. We define the restriction operator $\mathcal{R}_{h}$ from $V$ into $V_{h}$,

$$
\mathcal{R}_{h} u=\sum_{i=1}^{N} u\left(x_{i}\right) \chi_{i} \in V_{h}, \quad \forall u \in V .
$$

We note that this definition makes sense because $H_{0}^{1}(0,1) \subset \mathcal{C}[0,1]$. 
Remark 2.1. Some authors distinguish between finite difference (FD) and finite volume (FV) schemes by whether the discrete variables are defined as the pointwise values of the corresponding continuous variables (FD), or as the area/volume averaged values of the corresponding continuous variables $(\mathrm{FV})$; see [14. However, taking the discrete variable as the area/volume average of the continuous variable can lead to serious consistency issues, especially on non-uniform grids, as pointed out by [13. We follow the latter reference and distinguish FD and FV schemes by how the differential operators are discretized. If the differential operators are discretized by Green's or the divergence theorems, then the scheme is FV; if instead, all the individual derivatives are discretized by Taylor series expansion, then the scheme is FD. In fact, in most thus-defined FV schemes, the FD formulae are also used on certain parts of the equations, or on the fluxes that are part of the FV discretizations of the differential operators. For this reason, we sometimes call these schemes mixed finite difference finite volume schemes (FDFV, e.g. [23, 6]).

Remark 2.2. Our scheme, to be given later, will be classified as a finite difference scheme according to the definition adopted by [14], because the discrete variable is viewed as the pointwise value of the continuous variable (2.5). However, the one-dimensional grid that is used in this section is identical to the cell-centered FV grid, and differs from the FD grid in the same reference. Our own definition regrading FD and FV schemes, given in the preceding remark, does not make a difference on the problem under consideration in the one-dimensional space. Due to the technical differences with [14] in the meshes specifications and for the sake of completion, we provide full details on specifications and convergence analysis of the scheme below, while we acknowledge that these developments mostly parallel those in the cited reference, and differ in technical details. The error analysis part (Section 2.3) is new.

We define the gradient operators $\nabla_{h}$ for $V_{h}$,

$$
\nabla_{h} u_{h}=\sum_{i=0}^{N} \frac{u_{i+1}-u_{i}}{h_{i+\frac{1}{2}}} \chi_{i+\frac{1}{2}} \in \widetilde{V}_{h}, \quad \forall u \in V .
$$

The gradient operator is well-defined throughout the whole interval under the convention that

$$
u_{0}=u_{N+1}=0,
$$

which agrees with the homogeneous Dirichlet boundary conditions of $V$. The gradient operator $\widetilde{\nabla}_{h}$ on the dual function space $\widetilde{V}_{h}$ is defined as

$$
\widetilde{\nabla}_{h} \widetilde{u}_{h}=\sum_{i=1}^{N} \frac{\widetilde{u}_{i+\frac{1}{2}}-\widetilde{u}_{i-\frac{1}{2}}}{h_{i}} \chi_{i} \in V_{h}, \quad \forall \widetilde{u}_{h} \in V_{h} .
$$

The space $V_{h}$ is equipped with the semi- $H^{1}$ norm,

$$
\left\|u_{h}\right\|=\left|\nabla_{h} u_{h}\right|_{0} .
$$


In fact, due to the Poincaré inequality that we will establish later, $V_{h}$ is a Hilbert space under this norm.

Finally, we define the prolongation operator $\mathcal{P}_{h}$ from $V_{h}$ into $F$,

$$
\mathcal{P}_{h} u_{h}=\left(u_{h}, \nabla_{h} u_{h}\right) \in F, \quad \forall u_{h} \in V_{h} .
$$

For each $h \in \mathcal{H}$, the external approximation of the function space $V$ consists of the function space $F$, the isomorphic mapping $\Pi$, and the triplet $\left(V_{h}, \mathcal{R}_{h}, \mathcal{P}_{h}\right)$. Based on the specification of the norms of $V_{h}$ and $F$, it is straightforward to verify the following result.

Lemma 2.3. The prolongation operator $\mathcal{P}_{h}$ from $V_{h}$ into $F$ is stable.

In subsequent analyses, we will need the following discrete integration by parts formula,

Lemma 2.4. Let $u_{h} \in V_{h}, v_{h} \in \widetilde{V}_{h}$. Then

$$
\left(\nabla_{h} u_{h}, v_{h}\right)=-\left(u_{h}, \widetilde{\nabla}_{h} v_{h}\right) .
$$

Proof. By definition,

$$
\begin{aligned}
\left(\nabla_{h} u_{h}, v_{h}\right) & =\sum_{i=0}^{N} \frac{u_{i+1}-u_{i}}{h_{i+\frac{1}{2}}} v_{i+\frac{1}{2}} h_{i+\frac{1}{2}} \\
& =\sum_{i=0}^{N}\left(u_{i+1}-u_{i}\right) v_{i+\frac{1}{2}} .
\end{aligned}
$$

Using the conventions on the boundary terms $u_{0}$ and $u_{N+1}$, we can rewrite the summation as

$$
\begin{aligned}
\left(\nabla_{h} u_{h}, v_{h}\right) & =\sum_{i=1}^{N} u_{i}\left(v_{i-\frac{1}{2}}-v_{i+\frac{1}{2}}\right) \\
& =-\sum_{i=1}^{N} u_{i} \frac{v_{i+\frac{1}{2}}-v_{i-\frac{1}{2}}}{h_{i}} h_{i} \\
& =-\left(u_{h}, \widetilde{\nabla}_{h} v_{h}\right) .
\end{aligned}
$$

The convergence of external approximations to a function space has two conditions. The first states that, for any $u \in V, \mathcal{P}_{h} \mathcal{R}_{h} u$ should converge to $\Pi u$ under the strong topology of $F$. This condition ensures the consistency of the restriction and the projection operators. The other condition requires that if $\mathcal{P}_{h} u_{h}$ convergences weakly to $\omega$ in $F$, then $\omega$ is the image of an element of $V$. For the external approximation we have just constructed, the following lemma confirms that both of these convergence conditions are true.

Lemma 2.5. For the external approximation, the following hold true. 
(C1) For arbitrary $u \in V$,

$$
\mathcal{P}_{h} \mathcal{R}_{h} u \longrightarrow \Pi u \quad \text { strongly in } F \text {. }
$$

(C2) Let $\left\{u_{h}\right\}$ be a sequence in $F$. If

$$
\mathcal{P}_{h} u_{h} \rightarrow w \quad \text { weakly in } F,
$$

then, for some $u \in V$,

$$
w=\Pi u \text {. }
$$

Proof. For $(C 1)$,

$$
\begin{aligned}
\mathcal{R}_{h} u & =\sum_{i=1}^{N} u\left(x_{i}\right) \chi_{i}, \\
\mathcal{P}_{h} \mathcal{R}_{h} u & =\left(\mathcal{R}_{h} u, \nabla_{h} \mathcal{R}_{h} u\right) \\
& =\left(\sum_{i=1}^{N} u\left(x_{i}\right) \chi_{i}, \sum_{i=0}^{N} \frac{u\left(x_{i+1}\right)-u_{x_{i}}}{h_{i+\frac{1}{2}}} \chi_{i+\frac{1}{2}}\right) .
\end{aligned}
$$

As an easy application of the Taylor series expansion, it can be shown that, if $u \in \mathcal{D}(0,1)$, then

$$
\begin{array}{rlr}
\mathcal{R}_{h} u & \longrightarrow u, & \text { strongly in } L^{2}(0,1), \\
\nabla_{h} \mathcal{R}_{h} u & \longrightarrow u_{x}, & \text { strongly in } L^{2}(0,1) .
\end{array}
$$

Then the definition of $\mathcal{R}_{h}$ can be extended to the whole space $V$ and the above convergence still holds.

For $(C 2)$, the weak convergence

$$
\mathcal{P}_{h} u_{h} \rightarrow(u, w) \text { in } F
$$

implies that

$$
\begin{aligned}
u_{h} & \rightarrow u \text { in } L^{2}(0,1), \\
\nabla_{h} u_{h} & \rightarrow w \text { in } L^{2}(0,1) .
\end{aligned}
$$

Let $v \in \mathcal{D}(0,1)$, and $\widetilde{\mathcal{R}}_{h} v=\sum_{i=1}^{N} v\left(x_{i+\frac{1}{2}}\right) \chi_{i+\frac{1}{2}}$. It can be shown as in part (a) that

$$
\left(\widetilde{\mathcal{R}}_{h} v, \widetilde{\nabla}_{h} \widetilde{\mathcal{R}}_{h} v\right) \longrightarrow\left(v, v_{x}\right) \quad \text { strongly in } F .
$$

By the integration by parts formula, we have

$$
\left(\nabla_{h} u_{h}, \widetilde{\mathcal{R}}_{h} v\right)=-\left(u_{h}, \widetilde{\nabla}_{h} \widetilde{\mathcal{R}}_{h} v\right) .
$$

By the weak convergences in (2.11) - 2.12) and the strong convergences in (2.13), we obtain that

$$
(w, v)=-\left(u, v_{x}\right), \quad \text { for any } v \in \mathcal{D}(0,1) .
$$


This shows that

$$
\frac{\partial u}{\partial x}=w \quad \text { in } \mathcal{D}^{\prime}(0,1)
$$

Let $u^{\#}$ be the extension of $u$ by zero outside $(0,1)$, and let $w^{\#}$ be the extension of $w$ by zero outside $(0,1)$. It can be shown by the same procedure that

$$
\frac{\partial u^{\#}}{\partial x}=w^{\#} \quad \text { in } \mathcal{D}^{\prime}(0,1)
$$

Thus $u^{\#} \in H^{1}(\mathbb{R})$. The fact that $u^{\#}=0$ outside the interval $(0,1)$ indicates that $u^{\#}$ vanishes on the boundary. Thus,

$$
u=\left.u^{\#}\right|_{(0,1)} \in H_{0}^{1}(0,1)
$$

and the claim is proven.

We can now state the finite volume scheme for the 1D elliptic boundary value problem (2.1)-2.2):

For each $f \in L^{2}(0,1)$, let $f_{i}=\frac{1}{h_{i}} \int_{K_{i}} f d x$ and $f_{h}=\sum_{i=1}^{N} f_{i} \chi_{i}$.

Find $u \in V_{h}$ such that

$$
-\widetilde{\nabla}_{h} \nabla_{h} u_{h}=f_{h} .
$$

By the integration by parts formula (2.9), we obtain the variational form of the scheme,

$$
\left(\nabla_{h} u_{h}, \nabla_{h} v_{h}\right)=\left(f, v_{h}\right), \quad \forall v_{h} \in V_{h}
$$

2.2. Existence and uniqueness of a solution. We first establish the discrete Poincaré inequality.

Lemma 2.6. If $u_{h} \in V_{h}$, then

$$
\left|u_{h}\right|_{0} \leq C\left|\nabla_{h} u_{h}\right|_{0}
$$

for some $C>0$ independent of $h$.

Proof. The procedure mimics that for the continuous case. We let

$$
u_{h}=\sum_{i=1}^{N} u_{i} \chi_{i} .
$$

Then, by definition, we have

$$
\left|u_{h}\right|_{0}^{2}=\sum_{i=1}^{N} u_{i}^{2} h_{i} .
$$


For each individual $i, u_{i}$ can be expressed in terms of the differences,

$$
\begin{aligned}
u_{i} & =\sum_{j=1}^{i}\left(u_{j}-u_{j-1}\right) \\
& =\sum_{j=1}^{i} \frac{u_{j}-u_{j-1}}{h_{j-\frac{1}{2}}} h_{j-\frac{1}{2}} .
\end{aligned}
$$

Here, the convention that $u_{0}=0$ has been used. From this expression, and by the Cauchy-Schwarz inequality, we derive that

$$
\begin{aligned}
u_{i}^{2} & =\left(\sum_{j=1}^{i} \frac{u_{j}-u_{j-1}}{h_{j-\frac{1}{2}}} h_{j-\frac{1}{2}}\right)^{2} \\
& \leq \sum_{j=1}^{i}\left(\frac{u_{j}-u_{j-1}}{h_{j-\frac{1}{2}}}\right)^{2} h_{j-\frac{1}{2}} \cdot \sum_{j=1}^{i} h_{j-\frac{1}{2}} \\
& \leq \sum_{j=1}^{N+1}\left(\frac{u_{j}-u_{j-1}}{h_{j-\frac{1}{2}}}\right)^{2} h_{j-\frac{1}{2}} \cdot 1 \\
& =\left|\nabla_{h} u_{h}\right|_{0}^{2} .
\end{aligned}
$$

Thus, for the $L^{2}$-norm, we have

$$
\left|u_{h}\right|_{0}^{2} \leq\left|\nabla_{h} u_{h}\right|_{0}^{2} \sum_{i=1}^{N} h_{i}=\left|\nabla_{h} u_{h}\right|_{0}^{2} .
$$

Due to the discrete Poincaré inequality, the bilinear form $a_{h}(\cdot, \cdot)$ is coercive on $V_{h}$. Then the existence and uniqueness of a solution to the numerical scheme (2.18) follows from the Lax-Milgram theorem. An energy bound on the solution is given by the following

Lemma 2.7. Let $f \in L^{2}(0,1)$, and let $u_{h}$ be the corresponding discrete solution to the scheme (2.17). Then

$$
\left|u_{h}\right|_{1, h} \leq|f|_{0} .
$$

Proof. Replacing $v_{h}$ by $u_{h}$ in 2.18), and using the discrete Poincaré inequality, we have

$$
\begin{aligned}
\left|u_{h}\right|_{1, h}^{2} & =\left(\nabla_{h} u_{h}, \nabla_{h} u_{h}\right) \\
& =\left(f, u_{h}\right) \\
& \leq|f|_{0} \cdot\left|u_{h}\right|_{0} \\
& \leq|f|_{0} \cdot\left|u_{h}\right|_{1, h} .
\end{aligned}
$$

The claim 2.20 follows by dividing both sides by $\left|u_{h}\right|_{1, h}$. 
With the existence, uniqueness, and boundedness of the discrete solution, we can now show that the discrete solution converges, and the limit is a unique solution of the boundary value problem $(2.1)-2.2$.

Theorem 2.8. Let $f \in L^{2}(0,1)$, and let $u_{h}$ be the corresponding discrete solution of (2.17) (or (2.18)). Then there exists a unique $u \in V$ such that, as the mesh resolution refines,

$$
\mathcal{P}_{h} u_{h} \longrightarrow \Pi u \quad \text { strongly in } F,
$$

and $u$ solves the variational problem (2.3).

This result has been established in 14]. The proof given below follows the same ideas of the reference cited, but is adapted for the particular mesh specifications used here.

Proof. By the uniform bound 2.20 on $\left\{u_{h}\right\}_{h \in \mathcal{H}}$ and the weak compactness of $F$, there exists a subsequence $u_{h^{\prime}}$ such that

$$
\mathcal{P}_{h} u_{h^{\prime}} \equiv\left(u_{h^{\prime}}, \nabla_{h^{\prime}} u_{h^{\prime}}\right) \rightarrow(u, w) \quad \text { weakly in } F
$$

By the $(C 2)$ condition of Lemma 2.5, $u$ belongs to $V$, and

$$
\frac{d u}{d x}=w .
$$

Let $v \in \mathcal{D}(0,1)$ and $v_{h^{\prime}}=\mathcal{R}_{h^{\prime}} v$. Then by the $(C 1)$ condition of Lemma 2.5 ,

$$
\mathcal{P}_{h} \mathcal{R}_{h} v \equiv\left(R_{h} v, \nabla_{h} \mathcal{R}_{h} v\right) \longrightarrow \Pi v \equiv\left(v, v_{x}\right) \quad \text { strongly in } F .
$$

Since each $u_{h^{\prime}}$ is a solution to the scheme (2.18), and $v_{h^{\prime}} \in V_{h}$, we have

$$
\left(\nabla_{h^{\prime}} u_{h^{\prime}}, \nabla_{h^{\prime}} v_{h^{\prime}}\right)=\left(f, v_{h^{\prime}}\right) .
$$

Thanks to the weak convergence of $(2.22)$ and the strong convergence of (2.23), we can pass to the limit in (2.24) by sending $h^{\prime}$ to zero and obtain

$$
\left(u_{x}, v_{x}\right)=(f, v), \quad \forall v \in \mathcal{D}(0,1) .
$$

Since $\mathcal{D}(0,1)$ is a dense subspace of $V$, the above relation holds for every $v \in V$, and thus $u$ solves the variational equation (2.3).

The solution must be unique thanks to the coercivity of the bilinear operator $a(\cdot, \cdot)$.

Due to uniqueness, the whole sequence converges,

$$
\mathcal{P}_{h} u_{h} \equiv\left(u_{h}, \nabla_{h} u_{h}\right) \rightarrow\left(u, u_{x}\right) \quad \text { weakly in } F
$$

We now show that, actually, the convergence hold in the strong topology of $F$. We consider

$$
\begin{aligned}
\left|u_{h}-\mathcal{R}_{h} u\right|_{1, h}^{2} & =a_{h}\left(u_{h}-\mathcal{R}_{h} u, u_{h}-\mathcal{R}_{h} u\right) \\
& =\left(f, u_{h}\right)+\left(\nabla_{h} \mathcal{R}_{h} u, \nabla_{h} \mathcal{R}_{h} u\right)-\left(\nabla_{h} \mathcal{R}_{h} u, \nabla_{h} u_{h}\right)-\left(\nabla_{h} u_{h}, \nabla_{h} \mathcal{R}_{h} u\right)
\end{aligned}
$$


Thanks to the strong convergence in $(2.23)$ and the weak convergence in (2.26), we can pass to the limit in the above, and obtain

$$
\left|u_{h}-\mathcal{R}_{h} u\right|_{1, h}^{2}=(f, u)-\left(u_{x}, u_{x}\right)=0 .
$$

The conclusion (2.21) follows the observation that

$$
\begin{aligned}
\left|\mathcal{P}_{h} u_{h}-\Pi u\right|_{F} & =\left|\mathcal{P}_{h} u_{h}-\mathcal{P}_{h} \mathcal{R}_{h} u+\mathcal{P}_{h} \mathcal{R}_{h} u-\Pi u\right|_{H} \\
& \leq\left|\mathcal{P}_{h}\right| \cdot\left|u_{h}-\mathcal{R}_{h} u\right|_{V_{h}}+\left|\mathcal{P}_{h} \mathcal{R}_{h} u-\Pi u\right|_{F} .
\end{aligned}
$$

Remark 2.9. The current straightforward construction of $\mathcal{R}_{h}$ (see 2.5p) may NOT be extensible to high dimensional unstructured meshes, or to higherorder problems, e.g. $\Delta^{2}$.

2.3. Error estimates. We let $u$ be the true solution and $u_{h}$ be the solution of the numerical scheme (2.17) or 2.18). The discrete $H^{1}$-norm of the difference (error) can be written as

$$
\begin{aligned}
\left|u_{h}-\mathcal{R}_{h} u\right|_{1, h}^{2} & =a_{h}\left(u_{h}-\mathcal{R}_{h} u, u_{h}-\mathcal{R}_{h} u\right) \\
& =a_{h}\left(u_{h}, u_{h}-\mathcal{R}_{h} u\right)-a_{h}\left(\mathcal{R}_{h} u, u_{h}-\mathcal{R}_{h} u\right) .
\end{aligned}
$$

Since $u_{h}$ is a solution to the discrete scheme, we can use the relation (2.18) to rewrite the above expression as

$$
\left|u_{h}-\mathcal{R}_{h} u\right|_{1, h}^{2}=\left(f, u_{h}-\mathcal{R}_{h} u\right)-\left(\nabla_{h} \mathcal{R}_{h} u, \nabla_{h}\left(u_{h}-\mathcal{R}_{h} u\right)\right) .
$$

By the integration by parts formula $(2.9)$ on the second inner product, we obtain

$$
\left|u_{h}-\mathcal{R}_{h} u\right|_{1, h}^{2}=\left(f+\widetilde{\nabla}_{h} \nabla_{h} \mathcal{R}_{h} u, u_{h}-\mathcal{R}_{h} u\right) .
$$

On uniform meshes, we can use Taylor series expansion to show that the truncation error $f+\widetilde{\nabla}_{h} \nabla_{h} \mathcal{R}_{h} u$ converges to zero as fast as $O\left(h^{2}\right)$, and thus $\left|u_{h}-\mathcal{R}_{h} u\right|_{1, h}$ converges to zeros also at the second order. But this technique does not work for non-uniform mesh, because the discrete Laplace operators are often not consistent approximations to the corresponding differential operators $([13])$. To see that, we expand $u\left(x_{i \pm 1}\right.$ around $u\left(x_{i}\right)$ by Taylor series,

$$
\begin{aligned}
& u\left(x_{i+1}\right)=u\left(x_{i}\right)+h_{i+\frac{1}{2}} u_{x}\left(x_{i}\right)+\frac{1}{2} h_{i+\frac{1}{2}}^{2} u_{x x}\left(x_{i}\right)+O\left(h^{3}\right) \\
& u\left(x_{i-1}\right)=u\left(x_{i}\right)-h_{i-\frac{1}{2}} u_{x}\left(x_{i}\right)+\frac{1}{2} h_{i-\frac{1}{2}}^{2} u_{x x}\left(x_{i}\right)+O\left(h^{3}\right)
\end{aligned}
$$

Denoting $u\left(x_{i}\right)$ by $u_{i}$, etc., we obtain from these expansions that

$$
\begin{aligned}
& \frac{u_{i+1}-u_{i}}{h_{i+\frac{1}{2}}}=u_{x}\left(x_{i}\right)+\frac{1}{2} h_{i+\frac{1}{2}} u_{x x}\left(x_{i}\right)+O\left(h^{2}\right), \\
& \frac{u_{i}-u_{i-1}}{h_{i-\frac{1}{2}}}=u_{x}\left(x_{i}\right)-\frac{1}{2} h_{i-\frac{1}{2}} u_{x x}\left(x_{i}\right)+O\left(h^{2}\right) .
\end{aligned}
$$


Q. CHEN

Taking the difference between these two equations, and dividing both sides by $h_{i}$, we derive

$$
\left[\widetilde{\nabla}_{h} \nabla_{h} \mathcal{R}_{h} u\right]_{i} \equiv \frac{\frac{u_{i+1}-u_{i}}{h_{i+\frac{1}{2}}}-\frac{u_{i}-u_{i-1}}{h_{i-\frac{1}{2}}}}{h_{i}}=\frac{h_{i+\frac{1}{2}}+h_{i-\frac{1}{2}}}{2 h_{i}} u_{x x}\left(x_{i}\right)+O(h) .
$$

The truncation error for the discrete Laplace operator can be written as

$$
u_{x x}\left(x_{i}\right)-\left[\widetilde{\nabla}_{h} \nabla_{h} \mathcal{R}_{h} u\right]_{i}=\left(1-\frac{h_{i+\frac{1}{2}}+h_{i-\frac{1}{2}}}{2 h_{i}}\right) u_{x x}\left(x_{i}\right)+O(h) .
$$

Thus, on an non-uniform mesh, the discrete Laplace operator is a consistent approximation to the differential operator if and only if

$$
\frac{h_{i+\frac{1}{2}}+h_{i-\frac{1}{2}}}{2 h_{i}}=1 \text {. }
$$

This condition is generally not true on non-uniform meshes.

Instead of Taylor series expansion, we integrate (2.1) over $K_{i}$ to obtain

$$
-u_{x}\left(x_{i+\frac{1}{2}}\right)+u_{x}\left(x_{i-\frac{1}{2}}\right)=\left(f, \chi_{i}\right) .
$$

We define the restriction operator on the dual mesh as

$$
\widetilde{\mathcal{R}}_{h} u_{x}=\sum_{i=0}^{N} u_{x}\left(x_{i+\frac{1}{2}}\right) \chi_{i+\frac{1}{2}}
$$

We rewrite 2.29 as

$$
-\frac{u_{x}\left(x_{i+\frac{1}{2}}\right)-u_{x}\left(x_{i-\frac{1}{2}}\right)}{h_{i}} \cdot h_{i}=\left(f, \chi_{i}\right) .
$$

Using the newly defined restriction operator $\widetilde{\mathcal{R}}_{h}$, and the gradient operator $\widetilde{\nabla}_{h}$ on the dual mesh (see (2.7)), we can rewrite the above relation as

$$
-\left(\widetilde{\nabla}_{h} \widetilde{\mathcal{R}}_{h} u_{x}, \chi_{i}\right)=\left(f, \chi_{i}\right) .
$$

By integration by parts, it becomes

$$
\left(\widetilde{\mathcal{R}}_{h} u_{x}, \nabla_{h} \chi_{i}\right)=\left(f, \chi_{i}\right) .
$$

We note that this relation hold for arbitrary $1 \leq i \leq N$. Thus, for an arbitrary $v_{h} \in V_{h}$, the following relation holds,

$$
\left(\widetilde{\mathcal{R}}_{h} u_{x}, \nabla_{h} v_{h}\right)=\left(f, v_{h}\right)
$$

For arbitrary $x_{i+\frac{1}{2}}$, we note by Taylor series expansion that, if $u \in$ $C^{2}([0,1])$

$$
u^{\prime}\left(x_{i+\frac{1}{2}}\right)=\frac{u\left(x_{i+1}\right)-u\left(x_{i}\right)}{h_{i+\frac{1}{2}}}+\tau_{i+\frac{1}{2}}
$$


where $\tau_{i+\frac{1}{2}}$ represents the truncation error and

$$
\left|\tau_{i+\frac{1}{2}}\right| \leq C \cdot h
$$

We note that $u^{\prime}\left(x_{i+\frac{1}{2}}\right)$ represents the flux at $x_{i+\frac{1}{2}}$, which is the interface between cells $K_{i}$ and $K_{i+\frac{1}{2}}$ (Figure 1). Relation 2.33) indicates the consistency in the approximation to the Hux. Using the restriction operator $\mathcal{R}_{h}$ and the discrete gradient operator $\nabla_{h}$, we can rewrite 2.33) as

$$
\widetilde{\mathcal{R}}_{h} u_{x}=\nabla_{h} \mathcal{R}_{h} u+\tau_{h},
$$

with

$$
\tau_{h}=\sum_{i=0}^{N} \tau_{i+\frac{1}{2}} \chi_{i+\frac{1}{2}}, \quad\left|\tau_{h}\right|_{\infty} \leq C \cdot h .
$$

Thus 2.32 can be written as

$$
\left(\nabla_{h} \mathcal{R}_{h} u, \nabla_{h} v_{h}\right)=\left(f, v_{h}\right)-\left(\tau_{h}, v_{h}\right), \quad \forall v_{h} \in V_{h} .
$$

Using (2.35) in the relation (2.27), and by the Poincaré inequality, we obtain

$$
\begin{aligned}
\left|u_{h}-\mathcal{R}_{h} u\right|_{1, h}^{2} & =\left(\tau_{h}, u_{h}-\mathcal{R}_{h} u\right) \\
& \leq\left|\tau_{h}\right|_{0} \cdot\left|u_{h}-r_{h} u\right|_{0} \\
& \leq C\left|\tau_{h}\right|_{0} \cdot\left|u_{h}-\mathcal{R}_{h} u\right|_{1, h} .
\end{aligned}
$$

The estimate on the remainder term $\tau_{h}$ implies that

$$
\left|u_{h}-\mathcal{R}_{h} u\right|_{1, h} \leq C \cdot h \text {. }
$$

The $L^{2}$ error estimate can be trivially obtained through the Poincaré inequality,

$$
\left|u_{h}-\mathcal{R} u\right|_{0, h} \leq C\left|u_{h}-\mathcal{R}_{h} u\right|_{1, h} \leq C h .
$$

If $x_{i+\frac{1}{2}}$ is the midpoint of $K_{i+\frac{1}{2}}$, and $u \in \mathcal{C}^{3}([0,1])$, then the remainder $\tau_{h}$ is second-order in $h$, that is,

$$
\left|\tau_{h}\right|_{\infty} \leq C h^{2} .
$$

Then the semi $H^{1}$ and $L^{2}$ error estimates are of the second order as well,

$$
\begin{aligned}
& \left|u_{h}-\mathcal{R}_{h} u\right|_{1, h} \leq C \cdot h^{2}, \\
& \left|u_{h}-\mathcal{R}_{h} u\right|_{0, h} \leq C \cdot h^{2} .
\end{aligned}
$$

These results are summarized as follows.

Theorem 2.10. Let $u$ be the true solution of the elliptic boundary value problem (2.1) - (2.2), and $u_{h}$ be the discrete solution of the numerical scheme (2.18). If $u \in \mathcal{C}^{2}([0,1])$, then the following error estimates hold,

$$
\begin{aligned}
& \left|u_{h}-\mathcal{R}_{h} u\right|_{0, h} \leq C \cdot h, \\
& \left|u_{h}-\mathcal{R}_{h} u\right|_{1, h} \leq C \cdot h .
\end{aligned}
$$


If, furthermore, $x_{i+\frac{1}{2}}$ is the midpoint of $K_{i+\frac{1}{2}}$ for each $1 \leq i \leq N-1$, and $u \in \mathcal{C}^{3}([0,1])$, then the following error estimates hold,

$$
\begin{aligned}
& \left|u_{h}-\mathcal{R}_{h} u\right|_{0, h} \leq C \cdot h^{2}, \\
& \left|u_{h}-\mathcal{R}_{h} u\right|_{1, h} \leq C \cdot h^{2} .
\end{aligned}
$$

\section{The two-Dimensional InCOMPRESSible Stokes PRoblem}

3.1. Statement of the problem. We let $\Omega \subset \mathbb{R}^{2}$ be a bounded domain with smooth boundaries. The incompressible Stokes problem on this domain can be stated as follows,

$$
\begin{aligned}
-\Delta \boldsymbol{u}+\nabla p & =\boldsymbol{f}, & & \Omega \\
\nabla \cdot \boldsymbol{u} & =0, & & \Omega \\
\boldsymbol{u} & =0, & & \partial \Omega
\end{aligned}
$$

Here $\boldsymbol{u}=\boldsymbol{u}(x, y)$ is a two-dimensional vector field on $\Omega$ representing the velocity, $p=p(x, y)$ a scalar field representing the pressure, and $\boldsymbol{f}=\boldsymbol{f}(x, y)$ a two-dimensional vector field representing the external force. The boundary condition (3.3) is of the Dirichlet type. Using the vector identity

$$
\Delta \boldsymbol{u}=\nabla(\nabla \cdot \boldsymbol{u})+\nabla^{\perp}(\nabla \times \boldsymbol{u})
$$

and the incompressibility condition $(3.2)$, we obtain the vorticity formulation for equation (3.1),

$$
-\nabla^{\perp}(\nabla \times \boldsymbol{u})+\nabla p=\boldsymbol{f}, \quad \Omega .
$$

In the above, $\nabla^{\perp}=\mathbf{k} \times \nabla$ denotes the skewed gradient operator. Equations (3.5), (3.2) and (3.3) form the vorticity formulation of the two-dimensional incompressible Stokes problem. In this study, we use the vorticity formulation, as it highlights the role of vorticity, and seems most suitable for staggered-grid discretization techniques.

The natural functional space for the solution of the Stokes problem is

$$
V=\left\{\boldsymbol{u} \in H_{0}^{1}(\Omega) \mid \nabla \cdot \boldsymbol{u}=0 \text { in } \Omega\right\} .
$$

It is a Hilbert space under the norm

$$
\|\boldsymbol{u}\|_{V}=|\nabla \times \boldsymbol{u}|_{0} .
$$

By integration by parts, we obtain the weak formulation of the Stokes problem (3.5), 3.2)- (3.3):

For each $\boldsymbol{f} \in L^{2}(\Omega)$, find $\boldsymbol{u} \in V$ such that

$$
(\nabla \times \boldsymbol{u}, \nabla \times \boldsymbol{v})=(\boldsymbol{f}, \boldsymbol{v}), \quad \forall \boldsymbol{v} \in V .
$$

The existence and uniqueness of a solution to this problem follows from the Lax-Milgram theorem. 

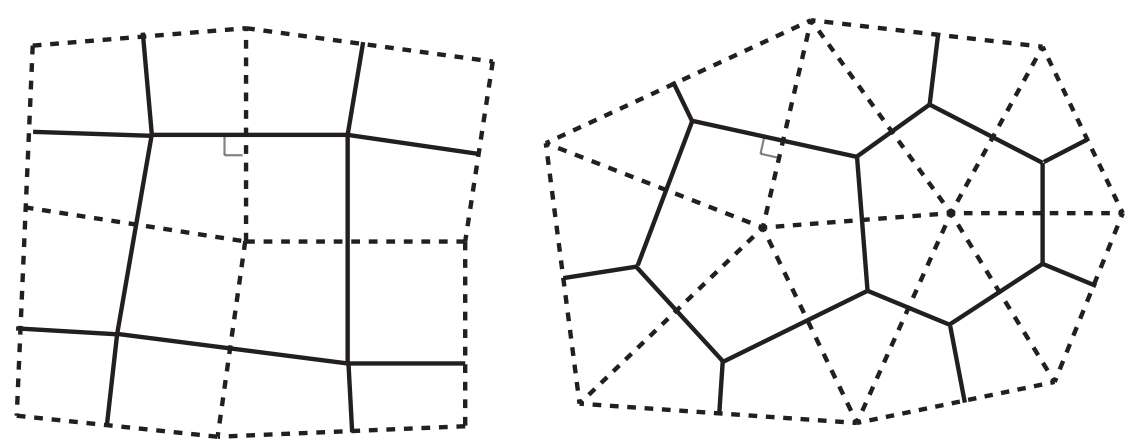

Figure 2. Generic dual meshes, with the domain boundaries passing through the primary cell centers. Left: a generic quadrilateral dual mesh. Right: a generic Delaunay-Voronoi dual mesh.

3.2. Mesh specifications and an external approximation of $V$. Our approximation of the function space is based on discrete meshes that consist of polygons. To avoid potential technical issues with the boundary, we shall assume that the domain $\Omega$ itself is polygonal. We make use of a pair of staggered meshes, with one called primary and the other called dual. The meshes consist of polygons, called cells, of arbitrary shape, but conforming to the requirements to be specified. The centers of the cells on the primary mesh are the vertices of the cells on the dual mesh, and vice versa. The edges of the primary cells intersect orthogonally with the edges of the dual cells. The line segments of the boundary $\partial \Omega$ pass through the centers of the primary cells that border the boundary. Thus the primary cells on the boundary are only partially contained in the domain. Two examples of this mesh type are shown in Figure 2.

TABLE 1. Sets of elements defining the connectivity of a unstructured dual grid.

\begin{tabular}{ll}
\hline Set & Definition \\
\hline $\mathrm{EC}(i)$ & Set of edges defining the boundary of primary cell $A_{i}$ \\
$\mathrm{VC}(i)$ & Set of dual cells that form the vertices primary cell $A_{i}$ \\
$\mathrm{CE}(e)$ & Set of primary cells boarding edge $e$ \\
$\mathrm{VE}(e)$ & Set of dual cells boarding edge $e$ \\
$\mathrm{CV}(\nu)$ & Set of primary cells that form vertices of dual cell $D_{\nu}$ \\
$\mathrm{EV}(\nu)$ & Set of edges that define the boundary of dual cell $D_{\nu}$ \\
\hline
\end{tabular}




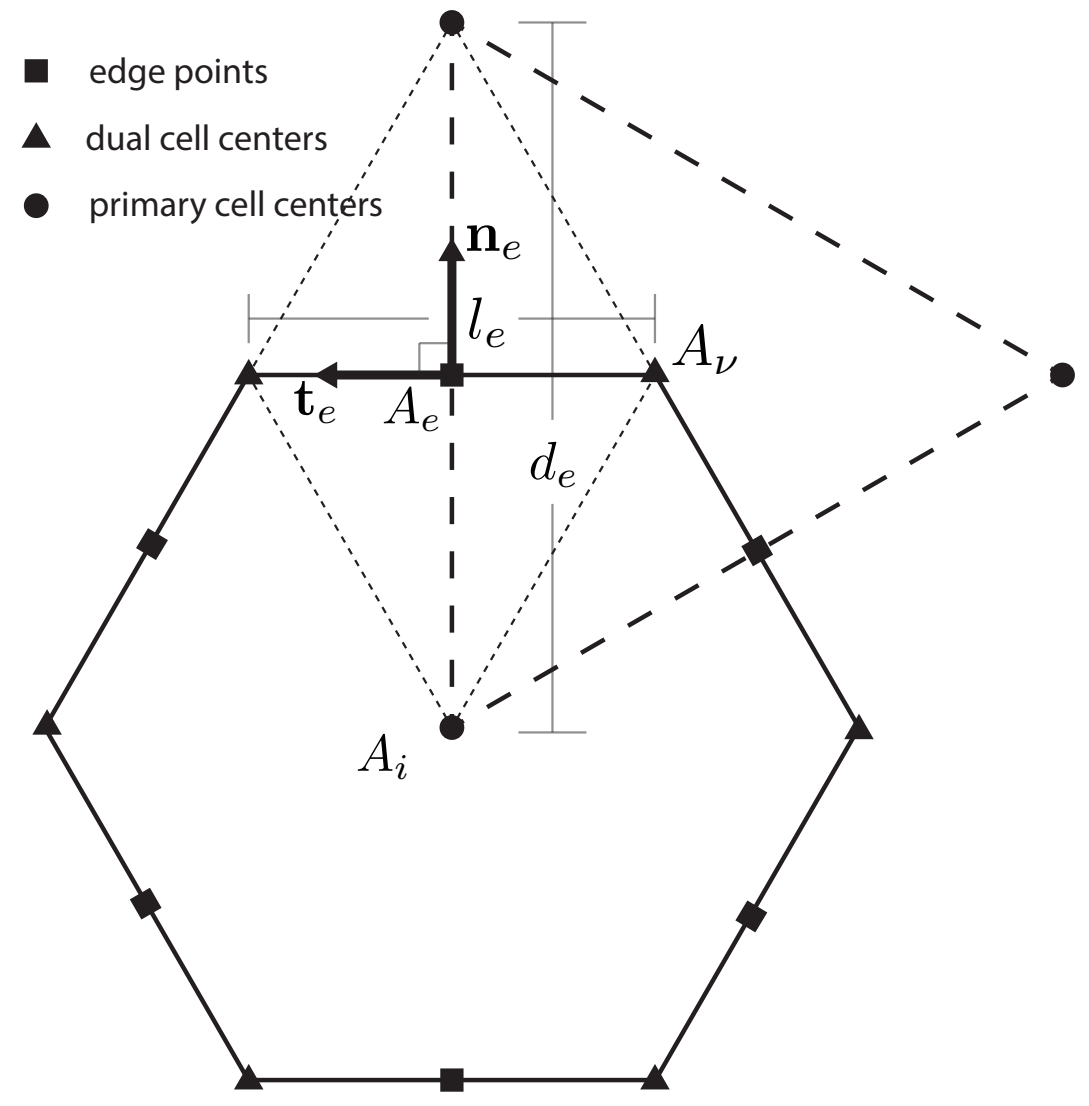

FiguRE 3. Notations

In order to construct function spaces on this type of meshes, some notations are in order, for which we follow the conventions made in [23, 6]. As shown in the diagram in Figure 3, the primary cells are denoted as $A_{i}, 1 \leq i \leq N_{c}+N_{c b}$, where $N_{c}$ denotes the number of cells that are in the interior of the domain, and $N_{c b}$ the number of cells that are on the boundary. We assume the cells are numbered so that $A_{i}$ with $1 \leq i \leq N_{c}$ refer to interior cells. The dual cells, which all lie inside the domain, are denoted as $A_{\nu}, 1 \leq \nu \leq N_{v}$. The area of $A_{i}$ (resp. $A_{\nu}$ ) is denoted as $\left|A_{i}\right|$ (resp. $\left|A_{\nu}\right|$ ). Each primary cell edge corresponds to a distinct dual cell edge, and vice versa. Thus the primary and dual cell edges share a common index $e, 1 \leq e \leq N_{e}+N_{e b}$, where $N_{e}$ denotes the number of edge pairs that lie entirely in the interior of the domain, and $N_{e b}$ the number of edge pairs on the boundary, i.e., with dual cell edge aligned with the boundary of the domain. Again, we assume that $1 \leq e \leq N_{e}$ refer to interior edges. Upon an edge pair $e$, the distance between the two primary cell centers, which is also the length of the corresponding dual cell edge, is denoted as $d_{e}$, while 
the distance between the two dual cell centers, which is also the length of the corresponding primary cell edge, is denoted as $l_{e}$. These two edges form the diagonals of a diamond-shaped region, whose vertices consist of the two neighboring primary cell centers and the two neighboring dual centers. The diamond-shaped region is also indexed by $e$, and will be referred to as $A_{e}$. The Euler formula for plannar graphs states that the number of primary cell centers $N_{c}+N_{c b}$, the number of vertices (dual cell centers) $N_{v}$, and the number of primary or dual cell edges $N_{e}+N_{e b}$ must satisfy the relation

$$
N_{c}+N_{c b}+N_{v}=N_{e}+N_{e b}+1 \text {. }
$$

The connectivity information of the unstructured staggered meshes is provided by six sets of elements defined in Table 1 .

For each edge pair, a unit vector $\mathbf{n}_{e}$, normal to the primary cell edge, is specified. A second unit vector $\mathbf{t}_{e}$ is defined as

$$
\mathbf{t}_{e}=\mathbf{k} \times \mathbf{n}_{e},
$$

with $\mathbf{k}$ standing for the upward unit vector. Thus $\mathbf{t}_{e}$ is orthogonal to the dual cell edge, but tangent to the primary cell edge, and points to the vertex on the left side of $\mathbf{n}_{e}$. For each edge $e$ and for each $i \in \mathrm{CE}(e)$ (the set of cells on edge $e$, see Table 1), we define the direction indicator

$$
n_{e, i}=\left\{\begin{aligned}
1 & \text { if } \mathbf{n}_{e} \text { points away from primary cell } A_{i}, \\
-1 & \text { if } \mathbf{n}_{e} \text { points towards primary cell } A_{i},
\end{aligned}\right.
$$

and for each $\nu \in \operatorname{VE}(e)$,

$$
t_{e, \nu}=\left\{\begin{aligned}
1 & \text { if } \mathbf{t}_{e} \text { points away from dual cell } A_{\nu}, \\
-1 & \text { if } \mathbf{t}_{e} \text { points towards dual cell } A_{\nu} .
\end{aligned}\right.
$$

For this study, we make the following regularity assumptions on the meshes. We assume that the diamond-shaped region $A_{e}$ is actually convex. In other words, the intersection point of each edge pair falls inside each of the two edges. We also assume that the meshes are quasi-uniform, in the sense that there exists $h>0$ such that, for each edge $e$,

$$
m h \leq l_{e}, d_{e} \leq M h
$$

for some fixed constants $(m, M)$ that are independent of the meshes. The staggered dual meshes are thus designated by $\mathcal{T}_{h}$. For the convergence analysis, it is assumed in [5] that, for each edge pair $e$, the primary cell edge nearly bisect the dual cell edge, and miss by at most $O\left(h^{2}\right)$. This assumption is also made here for the error analysis. Generating meshes conforming to this requirement on irregular domains, i.e. domains with non-smooth boundaries or domains on surfaces, can be a challenge, and will be addressed elsewhere. But we point out that, on regular domains with smooth boundaries, this type of meshes can be generated with little extra effort in addition to the use of standard mesh generators, such as the centroidal Voronoi tessellation algorithm ([11, 10, 9]). 
The approximation to the function space $V$ is given by

$$
V_{h}=\left\{u_{h}=\sum_{e=1}^{N_{e}} u_{e} \chi_{e} \mathbf{n}_{e} \mid \nabla_{h} \cdot u_{h}=0 .\right\}
$$

Thanks to the discrete Poincaré inequality for vector fields (see [5], $V_{h}$ is a Hilbert space under the norm

$$
\left\|u_{h}\right\|_{V_{h}} \equiv\left|u_{h}\right|_{1, h} \equiv\left|\widetilde{\nabla}_{h} \times u_{h}\right|_{0} .
$$

Since all functions in $V$ are divergence free, there is a one-to-one correspondence between $V$ and $H_{0}^{2}(\Omega)([17])$. We let

$$
F=L^{2}(\Omega) \times L^{2}(\Omega) .
$$

For each $\boldsymbol{u} \in V$, we let $\psi \in H_{0}^{2}(\Omega)$ and $\omega \in L^{2}(\Omega)$ such that

$$
\boldsymbol{u}=\nabla^{\perp} \psi, \quad \nabla \times \boldsymbol{u}=\omega .
$$

Then we redefine the isomorphism $\Pi$ from $V$ into $F$ as

$$
\Pi \boldsymbol{u}=(\psi, \omega) \in F, \quad \forall \boldsymbol{u} \in V .
$$

The space $F$ is endowed with the usual $L^{2}$-norm. In view of the norm for $V$, it is clear that $\Pi$ is an isomorphism from $V$ into $F$. It is important to note that the image of the operator $\Pi$ is not the whole space $F$. It is a nowhere dense, closed subspace of the latter.

Under the foregoing assumptions on the meshes, we now define the restriction operator $\mathcal{R}_{h}$. We only need to define $\mathcal{R}_{h}$ for a dense subset of functions of $V$, and the definition can then be extended to the whole space of $V$ according to a result in [24]. We let

$$
\mathcal{V}=\{\boldsymbol{u} \in \mathcal{D}(\Omega) \mid \nabla \cdot \boldsymbol{u}=0\},
$$

which is a dense subspace of $V$. For an arbitrary $\boldsymbol{u} \in \mathcal{V}$, there exists $\psi \in$ $\mathcal{D}(\Omega)$ such that $\boldsymbol{u}=\nabla^{\perp} \psi$, and thus $\omega=\Delta \psi$. We then define the associated discrete scalar field as

$$
\psi_{h}=\sum_{\nu=1}^{N_{v}} \psi_{\nu} \chi_{\nu}
$$

with

$$
\begin{array}{ll}
\psi_{\nu}=\psi\left(x_{\nu}\right), & \text { on interior dual cells, } \\
\psi_{\nu}=0, & \text { on dual cells on the boundary, }
\end{array}
$$

where $x_{\nu}$ is the coordinates for the center of dual cell $\nu$. We note that the function $\psi$ has compact support on $\Omega$, and therefore, if the grid resolution $h$ is fine enough, the specification (3.19) is consistent with (3.18). Finally, the restriction operator $\mathcal{R}_{h}$ on $\boldsymbol{u} \in \mathcal{V}$ is defined as

$$
\mathcal{R}_{h} \boldsymbol{u}=\widetilde{\nabla}_{h}^{\perp} \psi_{h} \text {. }
$$


$\mathcal{R}_{h} \boldsymbol{u}$ is divergence free in the discrete sense by construction (see Lemma 2.6 of [5]). It vanishes on the boundary thanks to the condition (3.19) and the definition for the skewed gradient operator on the boundary.

To define the prolongation operator $\mathcal{P}_{h}$, we note that, by the virtue of Lemma 2.6 of [5], every $u_{h} \in V_{h}$ is represented by a scalar field $\psi \in \Psi_{h}$ via

$$
u_{h}=\widetilde{\nabla}_{h}^{\perp} \psi_{h} .
$$

The prolongation operator $\mathcal{P}_{h}$ is defined as

$$
\mathcal{P}_{h} u_{h}=\left(\psi_{h}, \widetilde{\nabla}_{h} \times u_{h}\right), \quad \forall u_{h} \in V_{h} .
$$

The external approximation of $V$ consists of the mapping pair $(F, \Pi)$ and the family of triplets $\left\{V_{h}, \mathcal{R}_{h}, \mathcal{P}_{h}\right\}_{h \in \mathcal{H}}$. Concerning this approximation we have the following claim.

Theorem 3.1. The external approximation that consists of the function space $F$, the isomorphic mapping $\Pi$, and the family of triplets $\left\{V_{h}, \mathcal{R}_{h}, \mathcal{P}_{h}\right\}_{h \in \mathcal{H}}$ is a stable and convergent approximation of $V$.

The proof can be found in [5].

3.3. The MAC scheme. In discretizing the system, it is important to ensure that the external forcing $\boldsymbol{f}$ is also discretized in a consistent way. For the sake of simplicity in the convergence proof later on, we discretize the forcing term using its scalar stream and potential functions. For each $\boldsymbol{f} \in L^{2}(\Omega) \times L^{2}(\Omega)$, we let $\psi^{f} \in H_{0}^{1}(\Omega)$ and $\phi^{f} \in H^{1}(\Omega) / \mathbb{R}$ be such that

$$
\boldsymbol{f}=\nabla^{\perp} \psi^{f}+\nabla \phi^{f} .
$$

By the famous Helmholtz decomposition theorem, the stream and potential functions always exist and are unique, for each vector field $\boldsymbol{f}$ in $L^{2}(\Omega) \times L^{2}(\Omega)$ (see [17]). The stream and potential functions are discretized on the dual and primary meshes, respectively, by averaging,

$$
\begin{array}{rlrl}
\psi_{h}^{f} & =\sum_{\nu=1}^{N_{v}} \psi_{\nu}^{f} \chi_{\nu}, & \text { with } \psi_{\nu}^{f}=\bar{\psi}^{f} A_{\nu}, \\
\phi_{h}^{f}=\sum_{i=1}^{N_{c}+N_{c b}} \phi_{i}^{f} \chi_{i}, & \text { with } \phi_{i}^{f}=\bar{\phi}^{A_{i}} .
\end{array}
$$

Employing the technique of approximation by smooth functions and the Taylor's series expansion, we can show that the discrete scalar fields converge to the corresponding continuous fields in the $L^{2}$-norm, i.e.

$$
\begin{array}{ll}
\psi_{h}^{f} \longrightarrow \psi^{f} & \text { strongly in } L^{2}(\Omega), \\
\phi_{h}^{f} \longrightarrow \phi^{f} & \text { strongly in } L^{2}(\Omega) .
\end{array}
$$

With $\psi_{h}^{f}$ and $\phi_{h}^{f}$ defined as in (3.26) and (3.27), a discrete vector field can be specified,

$$
f_{h}=\widetilde{\nabla}_{h}^{\perp} \psi_{h}^{f}+\nabla_{h} \phi_{h}^{f}
$$


We take $f_{h}$ as the discretization of the continuous vector forcing field $f$. We should point out that this is not the only way to discretize the external forcing. There are ways that are probably more convenient in real applications, e.g. by taking $f_{h}$ to be the value of the normal component of $\boldsymbol{f}$ at the intersection points of the primary and dual cell edges. However, it will become clear later that the expression (3.28) is most convenient for convergence analysis. But other discretizations consistent with this one are also permissible.

The discrete problem can now be stated as follows.

For each $\boldsymbol{f} \in L^{2}(\Omega) \times L^{2}(\Omega)$, let $f_{h}$ be defined as in (3.28).

Find $u_{h} \in V_{h}$ and $p_{h} \in \Phi_{h}$ such that

$$
-\left[\widetilde{\nabla}_{h}^{\perp} \widetilde{\nabla}_{h} \times u_{h}\right]_{e}+\left[\nabla_{h} p_{h}\right]_{e}=f_{e}, \quad 1 \leq e \leq N_{e} .
$$

The incompressibility condition and the homogeneous boundary conditions on $u_{h}$ have been included in the specification of the space $V_{h}$. It is important to note that equation $(3.29)$ holds on interior edges only. On boundary edges, the computation of $\widetilde{\nabla}_{h}^{\perp} \widetilde{\nabla}_{h} \times u_{h}$ will require boundary conditions for $\widetilde{\nabla}_{h} \times u_{h}$, which are not available a priori.

As for the continuous problem, we multiply $(3.29)$ by $v_{h} \in V_{h}$ and integrate by parts, and noticing that $v_{h}=0$ along the boundary (see Lemma 2.5 of [5]), we obtain the variational form of the numerical scheme,

$$
\left(\widetilde{\nabla}_{h} \times u_{h}, \widetilde{\nabla}_{h} \times v_{h}\right)=2\left(f_{h}, v_{h}\right) .
$$

The term involving the pressure $p_{h}$ vanishes because of the incompressibility condition on $v_{h}$. The factor 2 on the right-hand side of (3.30) results from the integration-by-parts process. It can also be directly explained by the fact that the inner product on the right-hand side only involves the normal components of the vector fields. For $u_{h}, v_{h} \in V_{h}$, we define the bilinear form

$$
a_{h}\left(u_{h}, v_{h}\right)=\left(\widetilde{\nabla}_{h} \times u_{h}, \widetilde{\nabla}_{h} \times v_{h}\right) .
$$

Then the variational form of the numerical scheme can be stated as follows.

For each $\boldsymbol{f} \in L^{2}(\Omega) \times L^{2}(\Omega)$, let $f_{h}$ be defined as in (3.28).

Find $u_{h} \in V_{h}$ such that

$$
a_{h}\left(u_{h}, v_{h}\right)=2\left(f_{h}, v_{h}\right), \quad \forall v_{h} \in V_{h} .
$$

The existence and uniqueness of a solution $\boldsymbol{u}$ to the problem 3.6 follow from the Lax-Milgram theorem; such result can be found in [25]. The existence and uniqueness of a solution $u_{h}$ to the discrete scheme $(3.29)$ or equivalently (3.32), as well as its convergence to the true solution are presented in [5]; see also [12].

3.4. Error estimates. We let $\boldsymbol{u}$ be the true solution of $(3.1)-(3.3)$. For the purpose of error analysis, we assume that $\boldsymbol{u}$ is sufficiently smooth. The exact regularity requirements will be made explicit later. We let

$$
\omega=\nabla \times \boldsymbol{u}
$$


be the vorticity, which is also assumed to be sufficiently smooth. We then rewrite $(3.5)$ as

$$
-\nabla^{\perp} \omega+\nabla p=\boldsymbol{f} .
$$

We consider an interior edge pair $\left\{l_{e}, d_{e}\right\}$, with $l_{e}$ bounded by vertices $\nu_{1}$ and $\nu_{2}$, and $d_{e}$ bounded by cell centers $i_{1}$ and $i_{2}$ (Figure 3.4). The unit normal vector $\mathbf{n}_{e}$ on $l_{e}$ points from $i_{1}$ to $i_{2}$, and the unit tangential vector points from $\nu_{1}$ to $\nu_{2}$. Taking the inner product of (3.33) with $\mathbf{n}_{e}$ and averaging

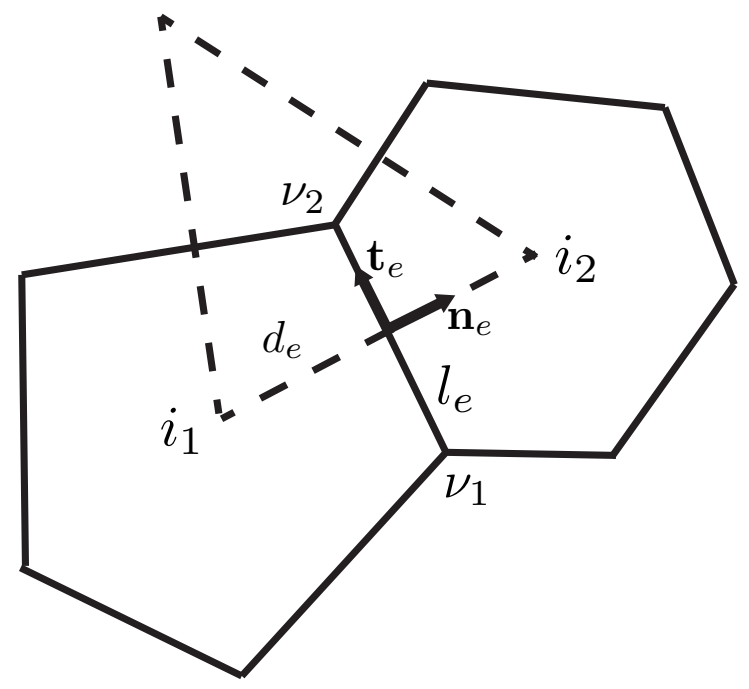

Figure 4. Edge pair

over $l_{e}$ yields

$$
\frac{\omega\left(x_{\nu_{2}}\right)-\omega\left(x_{\nu_{1}}\right)}{\left|l_{e}\right|}+\frac{1}{\left|l_{e}\right|} \int_{l_{e}} \frac{\partial p}{\partial n_{e}} d s=\frac{1}{\left|l_{e}\right|} \int_{l_{e}} \boldsymbol{f} \cdot \mathbf{n}_{e} d s .
$$

We note that, thanks to the fact that $l_{e}$ and $d_{e}$ nearly bisect each other, and provided that $p \in \mathcal{C}^{3}$, we have

$$
\frac{1}{\left|l_{e}\right|} \int_{l_{e}} \frac{\partial p}{\partial n_{e}} d s=\frac{p\left(x_{i_{2}}\right)-p\left(x_{i_{1}}\right)}{\left|d_{e}\right|}-\tau_{e}^{p},
$$

where

$$
\tau_{e}^{p}=O\left(h^{2}\right)
$$

is the truncation error for the pressure. From (3.23), $\boldsymbol{f}=\nabla^{\perp} \psi+\nabla \varphi$,

$$
\frac{1}{\left|l_{e}\right|} \int_{l_{e}} \boldsymbol{f} \cdot \mathbf{n}_{e} d s=\frac{1}{\left|l_{e}\right|} \int_{l_{e}}\left(-\frac{\partial \psi}{\partial t_{e}}+\frac{\partial \varphi}{\partial n_{e}}\right) d s .
$$

Again, by the fact that the dual cell edges $l_{e}$ and $d_{e}$ nearly bisect each other, and provided that $\psi^{f}$ and $\varphi^{f}$ are sufficiently smooth, the averaged quantities 
Q. CHEN

on the right-hand side can be approximate by the corresponding difference formulae to the second order, and therefore we have

$$
\frac{1}{\left|l_{e}\right|} \int_{l_{e}} \boldsymbol{f} \cdot \mathbf{n}_{e} d s=-\frac{\psi^{f}\left(x_{\nu_{2}}\right)-\psi^{f}\left(x_{\nu_{1}}\right)}{\left|l_{e}\right|}+\frac{\varphi^{f}\left(x_{c_{2}}\right)-\varphi^{f}\left(x_{c_{1}}\right)}{\left|d_{e}\right|}+\tau_{e}^{f},
$$

where $\tau_{e}^{f}$ is the local truncation error the $\boldsymbol{f}$, and

$$
\tau_{e}^{f}=O\left(h^{2}\right)
$$

We note that the external forcing $\boldsymbol{f}$ is discretized as

$$
f_{h}=\widetilde{\nabla}_{h}^{\perp} \psi_{h}+\nabla_{h} \varphi_{h} .
$$

Therefore, we have

$$
\frac{1}{\left|l_{e}\right|} \int_{l_{e}} \boldsymbol{f} \cdot \mathbf{n}_{e} d s=f_{e}+\tau_{e}^{f} .
$$

Combining (3.34), 3.35), and (3.37), we obtain

$$
\frac{\omega\left(x_{\nu_{2}}\right)-\omega\left(x_{\nu_{1}}\right)}{\left|l_{e}\right|}+\frac{p\left(x_{c_{2}}\right)-p\left(x_{c_{1}}\right)}{\left|d_{e}\right|}=f_{e}+\tau_{e}^{p}+\tau_{e}^{f} .
$$

Using the gradient and the skewed gradient operators, the above equation can be written as

$$
-\left[\widetilde{\nabla}_{h}^{\perp} \mathcal{R}_{h} \omega\right]_{e}+\left[\nabla_{h} \mathcal{R}_{h} p\right]_{e}=\left[f_{h}\right]_{e}+\tau_{e}^{p}+\tau_{e}^{f}, \quad 1 \leq e \leq N_{e} .
$$

We let $v_{h} \in V_{h}$ be arbitrary. Multiplying (3.39) by $v_{h}$ and integrating by parts yields the identity

$$
\left(\mathcal{R}_{h} \omega, \widetilde{\nabla}_{h} \times v_{h}\right)=2\left(f_{h}, v_{h}\right)+2\left(\tau_{h}^{p}+\tau_{h}^{f}, v_{h}\right) .
$$

It has been shown in [5] that the discrete curl of $\mathcal{R}_{h} u$ approximate $\mathcal{R}_{h} \omega$ with a first-order error, provided that $\psi^{u} \in \mathcal{C}^{3}(\Omega)$,

$$
\widetilde{\nabla}_{h} \times \mathcal{R}_{h} u=\mathcal{R}_{h} \omega+\tau_{h}^{\omega},
$$

where

$$
\tau_{h}^{\omega}=O(h) .
$$

This shows that the discrete curl operator $\widetilde{\nabla}_{h} \times(\cdot)$ is consistent with the curl operator $\nabla \times(\cdot)$. But, since the truncation error $\tau_{h}^{\omega}$ is only of the first order, the approximation to the Laplace operator, which is one order higher than the curl operator, will not be consistent, in general, on non-uniform meshes.

Substituting (3.41) into (3.40) yields

$$
\left(\widetilde{\nabla}_{h} \times \mathcal{R}_{h} u, \widetilde{\nabla}_{h} \times v_{h}\right)=2\left(f_{h}, v_{h}\right)+2\left(\tau_{h}^{p}+\tau_{h}^{f}, v_{h}\right)+\left(\tau_{h}^{\omega}, \widetilde{\nabla} \times v_{h}\right) .
$$

Thus the truncation error consists of three parts: the pressure gradient discretization error $\tau_{h}^{p}$, the external forcing discretization error $\tau_{h}^{f}$, and the vorticity discretization error $\tau_{h}^{\omega}$. 
Using the relation (3.32), the error in the discrete solution $u_{h}$ in the semi$H^{1}$ norm can be calculated as

$$
\begin{aligned}
\left|u_{h}-\mathcal{R}_{h} u\right|_{1}^{2} & =a_{h}\left(u_{h}-\mathcal{R}_{h} u, u_{h}-\mathcal{R}_{h} u\right) \\
& =a_{h}\left(u_{h}, u_{h}-\mathcal{R}_{h} u\right)-a_{h}\left(\mathcal{R}_{h} u, u_{h}-\mathcal{R}_{h} u\right) \\
& =2\left(f_{h}, u_{h}-\mathcal{R}_{h} u\right)-\left(\widetilde{\nabla}_{h} \times \mathcal{R}_{h} u, \widetilde{\nabla}_{h} \times\left(u_{h}-\mathcal{R}_{h} u\right)\right) .
\end{aligned}
$$

Using the relation (3.42), and the discrete Poincaré inequality, we obtain

$$
\begin{aligned}
\left|u_{h}-\mathcal{R}_{h} u\right|_{1}^{2} & =2\left(\tau_{h}^{p}+\tau_{h}^{f}, v_{h}\right)+\left(\tau_{h}^{\omega}, \widetilde{\nabla} \times v_{h}\right) \\
& \leq 2\left|\tau_{h}^{p}\right|_{0} \cdot\left|u_{h}-\mathcal{R}_{h} u\right|_{0}+2\left|\tau_{h}^{f}\right|_{0} \cdot\left|u_{h}-\mathcal{R}_{h} u\right|_{0}+\left|\operatorname{tau}_{h}^{\omega}\right|_{0} \cdot\left|u_{h}-\mathcal{R}_{h} u\right|_{1} \\
& \leq C\left(\left|\tau_{h}^{p}\right|_{0}+\left|\tau_{h}^{f}\right|_{0}+\left|\tau_{h}^{\omega}\right|_{0}\right) \cdot\left|u_{h}-\mathcal{R}_{h} u\right|_{1} .
\end{aligned}
$$

The estimate for the error in the $H^{1}$-norm thus results,

$$
\left|u_{h}-\mathcal{R}_{h} u\right|_{1} \leq C\left(\left|\tau_{h}^{p}\right|_{0}+\left|\tau_{h}^{f}\right|_{0}+\left|\tau_{h}^{\omega}\right|_{0}\right) \leq C h .
$$

In the above, $C$ is a constant independent of the grid resolution $h$ or the solution $u$. By another trivial application of the Poincaré inequality, the estimate for the $L^{2}$-norm of the error is obtained, which is of the same order.

The above result is formally summarized in

Theorem 3.2. If $\psi^{u} \in C^{3}(\Omega), \psi^{f} \in C^{3}(\Omega), \varphi^{f} \in C^{3}(\Omega)$, and $p \in C^{3}(\Omega)$, then the discrete solution $u_{h}$ is first-order accurate in the discrete $H^{1}$-norm, i.e.

$$
\left|u_{h}-\mathcal{R}_{h} u\right|_{1}=O(h) .
$$

It is worth noting that the first-order error in the discrete solution $u_{h}$ comes from the first-order error in the discretization of the vorticity, which is the Laplace of the streamfunction. On certain special meshes, such as the rectangular mesh, or the equilateral triangular mesh, it is well-known that the discretization error for the Laplace operator is second-order accurate. In these cases, the $H^{1}$-norm of the error in $u_{h}$ will also be of second order. However, at this point, we do not have a general characterization of meshes on which such result holds.

\section{Concluding Remarks}

Analysis of finite difference finite volume methods is hard and often adhoc, due to the lack of a variational framework; progresses have been made in this area by various authors $([14,15,8,16])$. Error analysis of FDFV methods is further hindered by the fact that FDFV schemes on non-uniform meshes are often inconsistent with the underlying differential equations. The present work adopts a new approach that combines the consistency in lowerorder differential operators with the external approximation framework for function spaces $([3,25])$. The new combined approach is first applied to 
Q. CHEN

the one-dimensional elliptic problem on a non-uniform mesh. A first-order convergence rate, in both $L^{2}$ and $H^{1}$ norms, is obtained, which agrees with the results obtained by a different approach in [13. The new combined approach is also applied to the MAC scheme for the incompressible Stokes problem on an unstructured mesh. A first-order convergence rate, in both $L^{2}$ and $H^{1}$ norms, is obtained. The results presented here improve those of Nicolaides ([22]) in that they hold on unstructured meshes. The current analysis also shows that, on certain special meshes, including the rectangular mesh considered by Nicolaides, the convergence rate is actually of the second order.

The new approach presented here has only a few general contingencies such as a stable and convergent external approximation of the function space associated with the continuous problem, and consistency in the approximation of the lower-order differential operators that appear in the equations. Thus, it is expected that the approach can be applied to derive error estimates for a wide range of problems, such as the steady-state Navier-Stokes problem, the time-dependent Navier-Stokes problem, the quasi-geostrophic equation for large-scale geophysical flows, and the shallow water equations. The present paper demonstrates the effectiveness of the new approach. Endeavors on the aforementioned systems is currently on-going, and the results will be presented elsewhere.

\section{REFERENCES}

1. K. Adamy and D. Pham, A finite volume implicit Euler scheme for the linearized shallow water equations: stability and convergence, Numer. Funct. Anal. Optim. 27 (2006), no. 7-8, 757-783.

2. A. Arakawa and V. R. Lamb, Computational design of the basic dynamical processes of the UCLA General Circulation Model, Methods Cornput, Methods in computational physics 17 (1977), 173-265.

3. Jean Céa, Approximation variationnelle des problèmes aux limites, Ann. Inst. Fourier (Grenoble) 14 (1964), no. fasc. 2, 345-444.

4. Qingshan Chen, On staggering techniques and the non-staggered Z-grid scheme, Numer. Math. 132 (2016), no. 1, 1-21.

5. __ Stable and convergent approximation of two-dimensional vector fields on unstructured meshes, Journal of Computational and Applied Mathematics 307 (2016), 284-306.

6. Qingshan Chen, Todd Ringler, and Max Gunzburger, A co-volume scheme for the rotating shallow water equations on conforming non-orthogonal grids, Journal of Computational Physics 240 (2013), 174-197.

7. S H Chou, Do Y Kwak, and Kwang Y Kim, A General Framework for Constructing and Analyzing Mixed Finite Volume Methods on Quadrilateral Grids: The Overlapping Covolume Case, SIAM Journal on Numerical Analysis 39 (2001), no. 4, 1170-1196.

8. Jerome Droniou, Robert Eymard, Thierry Gallouet, and Raphaele Herbin, Gradient schemes: a generic framework for the discretisation of linear, nonlinear and nonlocal elliptic and parabolic equations, Math. Models Methods Appl. Sci. 23 (2013), no. 13, 2395-2432.

9. Qiang Du, Vance Faber, and Max Gunzburger, Centroidal Voronoi tessellations: applications and algorithms, SIAM Rev. 41 (1999), no. 4, 637-676 (electronic). 
10. Qiang Du and Max Gunzburger, Grid generation and optimization based on centroidal Voronoi tessellations, Appl. Math. Comput. 133 (2002), no. 2-3, 591-607.

11. Qiang Du, Max D Gunzburger, and Lili Ju, Constrained centroidal Voronoi tessellations for surfaces, SIAM J. Sci. Comput. 24 (2003), no. 5, 1488-1506 (electronic).

12. Robert Eymard, Jürgen Fuhrmann, and Alexander Linke, On MAC schemes on triangular Delaunay meshes, their convergence and application to coupled flow problems, Numer. Methods Partial Differential Equations 30 (2014), no. 4, 1397-1424.

13. Robert Eymard, Thierry Gallouët, and Raphaèle Herbin, Finite volume methods, Handbook of numerical analysis, Vol. VII, North-Holland, Amsterdam, 2000, pp. 7131020.

14. S. Faure, D. Pham, and R. Temam, Comparison of finite volume and finite difference methods and application, Anal. Appl. (Singap.) 4 (2006), no. 2, 163-208.

15. Gung-Min Gie and Roger Temam, Cell centered finite volume methods using Taylor series expansion scheme without fictitious domains, Int. J. Numer. Anal. Model. 7 (2010), no. 1, 1-29.

16. Convergence of a cell-centered finite volume method and application to elliptic equations, Int. J. Numer. Anal. Model. 12 (2015), no. 3, 536-566.

17. V. Girault and P.A. Raviart, Finite element methods for Navier-Stokes equations: theory and algorithms, Springer-Verlag, Berlin, New York, 1986.

18. Francis H Harlow and Anthony A Amsden, Numerical calculation of almost incompressible flow, Journal of Computational Physics 3 (1968), no. 1, 80-93.

19. _ A numerical fluid dynamics calculation method for all flow speeds, Journal of Computational Physics 8 (1971), no. 2, 197-213.

20. Francis H Harlow and J Eddie Welch, Numerical Calculation of Time-Dependent Viscous Incompressible Flow of Fluid with Free Surface, Physics of Fluids 8 (1965), no. 12, 2182-2189.

21. Ronghua Li, Zhongying Chen, and Wei Wu, Generalized difference methods for differential equations, Monographs and Textbooks in Pure and Applied Mathematics, vol. 226, Marcel Dekker Inc., New York, 2000.

22. R. A. Nicolaides, Analysis and Convergence of the MAC Scheme I. The Linear Problem, SIAM Journal on Numerical Analysis 29 (1992), no. 6, pp. 1579-1591.

23. T. D. Ringler, J Thuburn, J. B. Klemp, and W. C. Skamarock, A unified approach to energy conservation and potential vorticity dynamics for arbitrarily-structured $C$-grids, Journal of Computational Physics 229 (2010), no. 9, 3065-3090.

24. R. Temam, Numerical Analysis, Kluwer-Springer-Verlag, 1980.

25. Navier-Stokes equations, AMS Chelsea Publishing, Providence, RI, 2001.

26. J Thuburn, TD Ringler, WC Skamarock, and JB Klemp, Numerical representation of geostrophic modes on arbitrarily structured $C$-grids, Journal of Computational Physics 228 (2009), no. 22, 8321-8335.

27. Pieter Wesseling, Principles of computational fluid dynamics, Springer Series in Computational Mathematics, vol. 29, Springer-Verlag, Berlin, Berlin, Heidelberg, 2001.

Department of Mathematical Sciences, Clemson University, Clemson, SC 29631, USA.

E-mail address: qsc@clemson.edu 